\title{
Comparing Charlson and Elixhauser comorbidity indices with different weightings to predict in-hospital mortality: an analysis of national inpatient data
}

\author{
Narayan Sharma ${ }^{1}$, René Schwendimann ${ }^{1,2}$ (D) Olga Endrich ${ }^{3}$ (D), Dietmar Ausserhofer ${ }^{1,4}$ (D) and Michael Simon ${ }^{1,5^{*}}$ (D)
}

\begin{abstract}
Background: Understanding how comorbidity measures contribute to patient mortality is essential both to describe patient health status and to adjust for risks and potential confounding. The Charlson and Elixhauser comorbidity indices are well-established for risk adjustment and mortality prediction. Still, a different set of comorbidity weights might improve the prediction of in-hospital mortality. The present study, therefore, aimed to derive a set of new Swiss Elixhauser comorbidity weightings, to validate and compare them against those of the Charlson and Elixhauser-based van Walraven weights in an adult in-patient population-based cohort of general hospitals.
\end{abstract}

Methods: Retrospective analysis was conducted with routine data of 102 Swiss general hospitals (2012-2017) for 6.09 million inpatient cases. To derive the Swiss weightings for the Elixhauser comorbidity index, we randomly halved the inpatient data and validated the results of part 1 alongside the established weighting systems in part 2, to predict in-hospital mortality. Charlson and van Walraven weights were applied to Charlson and Elixhauser comorbidity indices. Derivation and validation of weightings were conducted with generalized additive models adjusted for age, gender and hospital types.

Results: Overall, the Elixhauser indices, c-statistic with Swiss weights $(0.867,95 \% \mathrm{Cl}, 0.865-0.868)$ and van Walraven's weights $(0.863,95 \% \mathrm{Cl}, 0.862-0.864)$ had substantial advantage over Charlson's weights $(0.850,95 \% \mathrm{Cl}, 0.849-0.851)$ and in the derivation and validation groups. The net reclassification improvement of new Swiss weights improved the predictive performance by $1.6 \%$ on the Elixhauser-van Walraven and $4.9 \%$ on the Charlson weights.

Conclusions: All weightings confirmed previous results with the national dataset. The new Swiss weightings model improved slightly the prediction of in-hospital mortality in Swiss hospitals. The newly derive weights support patient population-based analysis of in-hospital mortality and seek country or specific cohort-based weightings.

Keywords: Comorbidity indices, Weights, Risk adjustment, Inpatient data, In-hospital mortality

\footnotetext{
* Correspondence: m.simon@unibas.ch

${ }^{1}$ Institute of Nursing Science (INS), Department Public Health (DPH), Faculty

of Medicine, University of Basel, Basel, Switzerland

${ }^{5}$ Nursing Research Unit, Inselspital University Hospital Bern, Bern, Switzerland

Full list of author information is available at the end of the article
}

\section{$\triangle B M C$}

(c) The Author(s). 2021 Open Access This article is licensed under a Creative Commons Attribution 4.0 International License, which permits use, sharing, adaptation, distribution and reproduction in any medium or format, as long as you give appropriate credit to the original author(s) and the source, provide a link to the Creative Commons licence, and indicate if changes were made. The images or other third party material in this article are included in the article's Creative Commons licence, unless indicated otherwise in a credit line to the material. If material is not included in the article's Creative Commons licence and your intended use is not permitted by statutory regulation or exceeds the permitted use, you will need to obtain permission directly from the copyright holder. To view a copy of this licence, visit http://creativecommons.org/licenses/by/4.0/ The Creative Commons Public Domain Dedication waiver (http://creativecommons.org/publicdomain/zero/1.0/) applies to the data made available in this article, unless otherwise stated in a credit line to the data. 


\section{Background}

Critical health outcomes such as mortality often require effective risk adjustment based on patient characteristics to predict in-hospital mortality. This is also true for comorbidities [1,2], which function as major predictors of mortality [3]. Over one-third of hospitalized patients have at least one comorbidity; two-thirds of those over $65[2,4]$ and three-quarters of those over 85 have at least two [5]. In addition to mortality, comorbidities are associated with lower health-related quality of life, increased disability and higher utilization of both health care services and prescribed medications [6-8].

Data on comorbidities are valuable both for comparison between patient populations and for risk adjustment regarding associated outcomes, especially mortality [9]. Two of the best-known measures are the Charlson Comorbidity Index and the Elixhauser Comorbidity Index $[10,11]$. When the Charlson Comorbidity Index was developed in 1987 it included 19 chronic conditions to predict one-year mortality, but has since been shortened to 17 . The Elixhauser Comorbidity Index, which was developed in 1998, works on a similar system but includes 30 - or, for some variants, 31 - comorbidities. In addition to in-hospital mortality, it is also used to predict the length of stay, adverse events and hospital discharges $[12,13]$. Despite this additional versatility (covering acute and chronic conditions) and strong evidence that the Elixhauser Comorbidity Index is statistically superior to the Charlson Comorbidity Index [13, 14], the Charlson Comorbidity Index continues to be used. Because of the fewer chronic conditions [15-17] and comparative ease of use in routine situations where time is limited.

Both indices work either via simple (unweighted) sum scores or as weighted scores assigning a risk weight to each comorbidity [6, 18, 19]. A weighted sum score/ summary measure provides an attractive advantage over plain dummy variables [20, 21], as it reduces the overfitting risk of more parameters, unjustifiable in small datasets [22] and limits computational requirements in large ones [21]. Additionally, evidence indicates that a weighted variable reduces type I errors compared to dummy variables while addressing multicollinearity concerns in regression analysis and organizing multiple highly correlated variables into more meaningful information [21, 23]. The weight assigned to each comorbidity reflects a higher, lower or neutral risk of mortality [24]. Practically, mortality risk scores can help to identify high-risk cases for special management and to assess provider services whose patients perform better or worse than expected from the summary measure of the morbidity burden.

To add to the value of early versions of the Elixhauser comorbidities, van Walraven et al. [25] used roughly 13 years' inpatient admission data from one Canadian hospital (1996-2008) to develop a set of weights (VW weights, i.e., the regression coefficient divided by the coefficient in the model with the smallest absolute value) for the 30 Elixhauser comorbidities associated with inhospital mortality. Using the backward selection and an alpha inclusion criterion of 0.05 to identify independently associated comorbidities, van Walraven identified 21 comorbidities significantly associated with mortality. A VW weight was assigned to each of the 21 Elixhauser comorbidities. Ultimately, VW weights ranged from -7 to 12 , with a weight of 0 assigned to the 9 nonsignificant comorbidities.

Since then, primarily in North America, studies have used VW weights to predict in-hospital mortality, especially in clearly defined patient groups such as surgical, orthopaedic, or cancer patients and those in single hospitals or intensive care unit (ICU) [12, 13, 21, 25, 26]. Moreover, the comorbidity weighting system might differ between all hospitalization and a restricted cohort; mortality and other outcomes; and between the countries [27]. Few studies have applied comorbidity adjustments to national or regional inpatient datasets $[21,28]$. Therefore, an analysis of a large heterogeneous patient population from a national dataset (Switzerland) is justified both to provide an overview of Elixhauser comorbidities in a European sample and potentially to optimize the comorbidity weights. In addition to increasing the generalizability of these comorbidity weights, the use of such a dataset, representing all hospital inpatient cases (i.e., hospitalisation episodes) from a large, heterogeneous patient population, would allow a very accurate comparison of weighting systems. Therefore, the aims of our study were 1) to derive a new Swiss comorbidity weighting on a national inpatient dataset to predict inhospital mortality; 2) to validate Charlson, Elixhauservan Walraven and new weights on a national inpatient dataset; and 3) to compare the predictive performance of in-hospital mortality of the three weighting systems.

\section{Methods \\ Study design and population}

This is a retrospective population-based analysis of 6 years' data (2012-2017) from the Swiss national inpatient dataset. Upon our application, subject to a data protection contract (as stipulated by article 22 of the Swiss Federal Act on Data Protection), the Swiss Federal Statistics Office (FSO) provided anonymized data from all Swiss hospital inpatient cases hospitalized between 2012 and 2017. This included not only general hospitals but also special care (e.g., paediatric, gynaecological) facilities [29]. The FSO classifies general hospitals (University hospitals, Tertiary hospitals, and three Basic hospitals) into five different levels, based on the number 
of cases treated per year and/or a special hospital score assigned by Swiss Medical Association ("FMH-Kategorien"). For this study, special care hospitals and children were excluded because of the low levels of comorbidities and the relatively low risk of dying in the hospital [25]. For data protection reasons, age was grouped in fiveyear groups, and all inpatient cases below 20 years of age were excluded. The flowchart for the final adult population included 102 general hospitals (6,094,672 inpatient cases) for the analysis is reported in supplementary Fig. F1 (Additional file 1).

\section{Dataset and classification of comorbidities indices}

The dataset included patient characteristics including sex, age, hospital types, primary and secondary diagnoses based on International Classification of Diseases-10 (ICD-10) codes and hospital discharge information including in-hospital mortality. As condition coding in Switzerland is based on the ICD-10 German Modification (ICD-10 GM), reported in supplementary Table S1 (Additional file 1), we used this to identify both Charlson and Elixhauser comorbidities. Specifically, we used Quan et al.'s ICD-10 codes [19] to determine each of the 17 Charlson and 31 Elixhauser comorbidities via the "Comorbidity" package in R [18]. This transforms ICD-10 codes into binary data the relevant comorbidities, their (unweighted) sum scores, and their Charlson and VWweighted scores.

\section{Descriptive analysis}

The study population's general characteristics (hospital types, patient's sex, and age groups) were reported in the alive and mortality cohorts with percentages. The distributions of Charlson and Elixhauser comorbidities, unweighted and weighted scores were computed as percentages of index values of $0,1-2$, and $\geq 3$ and $<0,0$, $1-4$, and $\geq 5$; and as the Charlson weight do not use negative weightings, its weights were calculated for index values of $0,1-4$, and $\geq 5$. For each characteristic and comorbidities, standardized mean differences (SMD) between the alive and mortality cohort were computed using "tableone" package in R. The SMD is identical to Cohen's D and provides an effect size estimate less sensitive to the sample size than $p$ values between the cohorts. This is important in a dataset of the size used in this study. An SMD of zero means there is no difference in the characteristics (e.g., gender) between the alive and mortality cohort. SMDs greater than 0.1 indicate potentially relevant differences [30], i.e., showing unbalanced covariates and might have an association with mortality.

\section{Derivation of Swiss comorbidity weights}

The study population was randomly split into a derivation $(50 \%)$ and a validation $(50 \%)$ group. The derivation group was used to determine the adjusted association of all 31 Elixhauser comorbidities with death, treating the anonymous hospital identifier as a random effect [31]. Generalized additive regression models (GAM) can accommodate many predictors including random effect, able to handle large dataset easily and nonparametric in nature [32]. We fitted GAM to compute the odds ratios (OR) using the package "mgcv" [33] and R programming language, version 3.5.2 [34]. We utilized GAM with random effect components on the hospital level, as university and small hospitals are different in size and services in Switzerland. To identify Elixhauser comorbidities associated with in-hospital mortality, we retained variables based on an alpha inclusion criterion of 0.01 .

To derive the Swiss weightings from the regression model's parameter estimates, we used the method described by Sullivan et al. [35]. Comorbidities not significantly associated with mortality were assigned a weight of zero. The number of (weighted) points assigned to each comorbidity equalled its regression coefficient divided by the coefficient in the model with the smallest absolute value [14, 21, 25, 35] rounded to the nearest whole number. Each person's new Elixhauser comorbidity weighting score was then calculated by summing up all points of all their coded comorbidities.

\section{Validation and comparison of weighted comorbidity models}

To validate and compare the performance of the three comorbidity weighting systems, we first created four multivariate in-hospital mortality prediction GAMs for the derivation group. The first model, 'base', contained no comorbidity data - only age group, sex, and hospital types. The other three models used the same variables as the base model, with the first, 'Charlson', using Charlson weights, the second, 'van Walraven', using the Elixhauser index with van Walraven weights, and the third, 'Swiss weights', using our newly-developed weights. We then validated all weights in validation groups by splitting the validation group into six groups by year of discharge. Altogether, 24 c statistics (including base models) were computed to validate the Charlson, van Walraven, and Swiss weights models in the validation sample. An additional four models were created using all cases (combining derivation and validation groups) to evaluate the performance of each model in the total patient population.

We assessed the various comorbidity weightings according to the model performance criteria. Discrimination, i.e., each model's ability to distinguish patients discharged alive from those who died in hospital, was compared using the concordance (c) statistic. The cstatistic quantified each model's ability to assign high probabilities of mortality to patients who died [36]. It's 
possible values range from 0.50 to 1.0 , with 0.50 indicating no ability to discriminate, values less than 0.70 are considered poor, those between 0.70 and 0.80 acceptable, and those of 0.80 or above excellent [37]. Using bootstrap methods, we computed $95 \%$ confidence intervals for each c-statistic. Additionally, the observed value was also explored for each model from the predicted values to observe the model performances in the highest selected percentages (1, 2, 5 and 10\%) in the derivation sample. We also graphed receiver-operating characteristic curves (ROC) for the visual presentation of the derivation group's c statistics. We compared the base model and existing comorbidity models with Swiss weight model using net reclassification improvement (NRI) for binary outcome [38-40] from the "nricens" package in R [41] using the Swiss derivation sample and classification cut-off value of 0.023 (mortality proportion of the total study population). NRI measures the degree of improvement in predicted inpatient mortality probabilities when comorbidity weights are added to the base model [21, 42]. Higher NRI values indicate more accurate reclassification.

\section{Code validation and sensitivity analyses}

We also evaluated the $\mathrm{R}$ comorbidity package's code handling accuracy in the Swiss setting. To do so we sampled 100 cases and manually reviewed the Swiss ICD-10 codes of the raw data, checking whether the "comorbidity" package had assigned each to the appropriate Charlson or Elixhauser comorbidity. We also performed sensitivity analyses to explore Switzerland's Major Diagnostic Categories' (MDCs') associations, which are based on ICD-10 GM (one way of expressing the reason for admission), if any, regarding the change in the predictability of in-hospital mortality in combination with the above models and to test whether the combined models' patterns differed from those of uncombined ones. MDCs are 24 mutually exclusive categories into which all primary diagnoses are assigned based on the Swiss diagnostic-related group (DRG) system for hospital reimbursement [43].

\section{Results}

\section{Population characteristics}

Overall, the adult inpatient population between 2012 and 2017 in all Swiss general hospitals (102) consisted of $6,094,672$ cases. Among all hospitalized cases in our study population mortality was $2.3 \%$. The characteristics of the adult inpatient cases are presented in Table 1. Inpatient cases had between 0 and 9 Charlson comorbidities (median 0, interquartile range (IQR): $0-1$ ) and between 0 and 16 Elixhauser comorbidities (median 1, IQR: 0-2). The different categories of three comorbidity weightings are presented in supplementary Table S2 (Additional file 1).

\section{Prevalence of Charlson and Elixhauser comorbidity indices}

The most common Charlson comorbidity was any malignancy (including lymphoma and leukaemia, except malignant neoplasm of the skin) in both cohorts, alive $(10.2 \%)$ and mortality (37.6\%), yet with marked differences between the two cohorts (SMD: 0.680). The prevalence for each Charlson comorbidity in the total population and the derivation is presented in supplementary Table S3 (Additional file 1).

The most common Elixhauser comorbidities were uncomplicated hypertension (22.7\%) in the alive cohort, whereas in the mortality cohort, it was solid tumour without metastasis (33.7\%). However, the most pronounced difference between both cohorts was observed for metastatic cancer (4.0\% vs. $26.5 \%$; SMD: 0.657$)$. The prevalence for each Elixhauser comorbidity from the total population and derivation group is presented in the supplementary Table S4 (Additional file 1).

\section{Derivation of Swiss weights}

In the derivation group, two of the 31 Elixhauser comorbidities showed no association with hospital mortality and were removed, leaving 29 in the final model with random effect on the hospital level. Sixteen were associated with increased mortality risk, with the strongest associations coming from metastatic cancer (OR: 4.09, 95\% CI: 3.98-4.21) and liver disease (OR: 3.83, 95\% CI: 3.70-3.97). At the other end of the spectrum, 13 comorbidities were associated with a decreased risk of hospital mortality. The strongest of these were deficiency anaemia (OR: 0.54, 95\% CI: 0.51-0.56) and obesity (OR: 0.59, 95\% CI: 0.56-0.63). The adjusted coefficients were used to derive Swiss weights with a new maximum weight of 17 , for metastatic cancer, and a new minimum of -7 , for deficiency anaemia (Table 2 ).

\section{Validation and comparison of weighted comorbidity models}

All three comorbidity weighting systems (Charlson, Elixhauser van Walraven and Swiss) indicated higher inhospital mortality risk than the base model, showing the conditional interpretation of weights for each of the weighted models. Each model performed similarly across all years in validation groups as in the derivation groups. Overall, the c-statistic for the 6-year cohort were: 0.757 (95\% CI: 0.755-0.759) for the base model, 0.850 (95\% CI: $0.849-0.851$ ) for Charlson, 0.863 (95\% CI: $0.862-$ 0.864 ) for VW Elixhauser and 0.867 (95\% CI: $0.865-$ 0.868 ) with Swiss Elixhauser. These c-statistics were similar in the development and validation cohorts. All 
Table 1 General characteristics of the total study population

\begin{tabular}{|c|c|c|c|}
\hline Parameters & Alive cohort (\%) & Mortality cohort (\%) & SMD \\
\hline aTotal population: $N=6,094,672$ & $5,952,005(97.7)$ & $142,667(2.3)$ & \\
\hline Females & $3,280,823(55.1)$ & $63,912(44.8)$ & 0.208 \\
\hline Age groups & & & 1.006 \\
\hline $20-24$ years & $215,672(3.6)$ & $292(0.2)$ & \\
\hline $25-29$ years & $327,562(5.5)$ & $375(0.3)$ & \\
\hline $30-34$ years & $415,022(7.0)$ & $526(0.4)$ & \\
\hline $35-39$ years & $348,591(5.9)$ & $718(0.5)$ & \\
\hline 40-44 years & $299,985(5.0)$ & $1368(1.0)$ & \\
\hline 45-49 years & $350,899(5.9)$ & $2503(1.8)$ & \\
\hline $50-54$ years & $408,028(6.9)$ & $4312(3.0)$ & \\
\hline $55-59$ years & $430,721(7.2)$ & $6503(4.6)$ & \\
\hline $60-64$ years & $466,543(7.8)$ & $9068(6.4)$ & \\
\hline $65-69$ years & $528,374(8.9)$ & $13,322(9.3)$ & \\
\hline 70-74 years & $554,612(9.3)$ & $16,899(11.8)$ & \\
\hline $75-79$ years & $535,543(9.0)$ & $19,888(13.9)$ & \\
\hline $80-84$ years & $509,225(8.6)$ & $24,853(17.4)$ & \\
\hline $85-89$ years & $365,924(6.1)$ & $24,042(16.9)$ & \\
\hline 90-94 years & $161,236(2.7)$ & $14,156(9.9)$ & \\
\hline $95+$ years & $34,068(0.6)$ & $3842(2.7)$ & \\
\hline Hospital types & & & 0.157 \\
\hline University (level 1) & $1,078,612(18.1)$ & $29,379(20.6)$ & \\
\hline Tertiary care (level 2) & $3,274,382(55.0)$ & $83,686(58.7)$ & \\
\hline Basic care (level 3) & $736,465(12.4)$ & $14,863(10.4)$ & \\
\hline Basic care (level 4) & $671,182(11.3)$ & $10,695(7.5)$ & \\
\hline Basic care (level 5) & $191,364(3.2)$ & $4044(2.8)$ & \\
\hline Number of Charlson comorbidities & & & 1.234 \\
\hline 0 & $3,642,650(61.2)$ & $17,465(12.2)$ & \\
\hline $1-2$ & $1,907,761(32.1)$ & $80,876(56.7)$ & \\
\hline$>=3$ & $401,594(6.7)$ & $44,326(31.1)$ & \\
\hline Number of Elixhauser comorbidities & & & 1.039 \\
\hline 0 & $2,509,169(42.2)$ & $11,036(7.7)$ & \\
\hline $1-2$ & $2,106,780(35.4)$ & $43,494(30.5)$ & \\
\hline$>=3$ & $1,336,056(22.4)$ & $88,137(61.8)$ & \\
\hline
\end{tabular}

Abbreviations: SMD standardized mean difference between alive and mortality cohort

${ }^{\mathrm{a}}$ Total population presented in row percentage

differences and the rankings they established among models were statistically significant. (Additional file 1, Table S5). In comparison, the model with Swiss weights discrimination was slightly better with some c-statistic variability across the 6 years' data.

Additionally, $1 \%$ highest predicted value, showed the same order of the model's performance from the observed mortality (base: 10.7\%, Charlson: 18.5\%, VW Elixhauser: 20.4\%, Swiss Elixhauser: 20.9\% (Table S6, Additional file 1). As shown in receiver-operating characteristic (ROC) curves (Fig. 1) the Swiss weights model's discrimination was better than the Charlson's or base model's, and only slightly better than the van Walraven's. The NRI confirm this picture (Table 3). Comparing the Swiss weights with VW weights showed an NRI of $1.6 \%$ (95\%-CI: 1.3-2.0) with differences in predicted probabilities of mortality (among those who died) of $1.4 \%$ and differences in predicted probabilities of alive (among those who lived) by $0.02 \%$.

Finally, the sensitivity analysis using MDCs did not offer any improvements in the models' performance. 
Table 2 Prevalence, adjusted odds ratio and weights from the (new) Swiss derivation sample and the van Walraven (WW) derivation sample [25]

\begin{tabular}{|c|c|c|c|c|c|c|c|}
\hline \multirow[t]{2}{*}{ Elixhauser comorbidities } & Alive cohort (\%) & Mortality cohort (\%) & \multirow[t]{2}{*}{ SMD } & \multicolumn{2}{|c|}{ Adjusted odds ratio $(95 \% \mathrm{Cl})$} & \multicolumn{2}{|c|}{ Weights } \\
\hline & \multicolumn{2}{|c|}{ Swiss derivation sample } & & $\mathrm{VW}^{\mathrm{a}}$ & Swiss & $\mathrm{VW}^{\mathrm{a}}$ & Swiss \\
\hline${ }^{\mathrm{b}}$ Derivation group & $2,975,887(97.7)$ & $71,449(2.3)$ & & & & & \\
\hline Congestive heart failure & $163,685(5.5)$ & $16,333(22.9)$ & 0.514 & $1.96(1.85-2.07)$ & $3.07(3.00-3.14)$ & 7 & 13 \\
\hline Cardiac arrhythmias & $341,280(11.5)$ & $20,754(29.0)$ & 0.448 & $1.71(1.62-1.80)$ & $1.69(1.66-1.73)$ & 5 & 6 \\
\hline Valvular disease & $117,450(3.9)$ & $6568(9.2)$ & 0.213 & $0.91(0.82-0.99)$ & $0.92(0.89-0.95)$ & -1 & -1 \\
\hline Pulmonary circulation disorders & $53,292(1.8)$ & $4813(6.7)$ & 0.247 & $1.48(1.34-1.62)$ & $1.62(1.57-1.68)$ & 4 & 6 \\
\hline Peripheral vascular disorders & $141,051(4.7)$ & $6912(9.7)$ & 0.192 & $1.26(1.17-1.36)$ & $1.27(1.24-1.31)$ & 2 & 3 \\
\hline Hypertension (uncomplicated) & $676,609(22.7)$ & $15,692(22.0)$ & 0.019 & - & $0.69(0.68-0.70)$ & 0 & -4 \\
\hline Hypertension (complicated) & $218,656(7.3)$ & $11,003(15.4)$ & 0.256 & - & $0.79(0.77-0.81)$ & 0 & -3 \\
\hline Paralysis & $61,546(2.1)$ & $5153(7.2)$ & 0.246 & $1.93(1.75-2.12)$ & $2.60(2.52-2.69)$ & 7 & 11 \\
\hline Other neurological disorders & $120,045(4.0)$ & $8011(11.2)$ & 0.273 & $1.83(1.70-1.96)$ & $2.45(2.39-2.52)$ & 6 & 10 \\
\hline Chronic pulmonary disease & $170,770(5.7)$ & $8269(11.6)$ & 0.209 & $1.36(1.29-1.44)$ & $1.31(1.27-1.34)$ & 3 & 3 \\
\hline Diabetes, uncomplicated & $245,817(8.3)$ & $9059(12.7)$ & 0.145 & - & $1.09(1.06-1.11)$ & 0 & 1 \\
\hline Diabetes, complicated & $66,161(2.2)$ & $2763(3.9)$ & 0.096 & - & $0.89(0.86-0.93)$ & 0 & -1 \\
\hline Hypothyroidism & $126,062(4.2)$ & $3454(4.8)$ & 0.029 & - & $0.76(0.74-0.79)$ & 0 & -3 \\
\hline Renal failure & $289,047(9.7)$ & $20,526(28.7)$ & 0.497 & $1.63(1.54-1.73)$ & $2.06(2.02-2.11)$ & 5 & 8 \\
\hline Liver disease & $49,916(1.7)$ & $5822(8.1)$ & 0.303 & $2.97(2.73-3.22)$ & $3.83(3.7-3.97)$ & 11 & 16 \\
\hline Peptic ulcer disease, excluding bleeding & $5808(0.2)$ & $258(0.4)$ & 0.032 & - & - & 0 & 0 \\
\hline AIDS/HIV & $2300(0.1)$ & $85(0.1)$ & 0.013 & - & - & 0 & 0 \\
\hline Lymphoma & $25,049(0.8)$ & $1759(2.5)$ & 0.127 & $2.55(2.31-2.81)$ & $2.19(2.07-2.31)$ & 9 & 9 \\
\hline Metastatic cancer & $119,667(4.0)$ & $18,907(26.5)$ & 0.657 & $3.30(3.10-3.52)$ & $4.09(3.98-4.21)$ & 12 & 17 \\
\hline Solid tumour without metastasis & $268,298(9.0)$ & $24,046(33.7)$ & 0.631 & $1.47(1.39-1.56)$ & $2.36(2.3-2.42)$ & 4 & 10 \\
\hline Rheumatoid arthritis/collagen vascular diseases & $47,305(1.6)$ & $1254(1.8)$ & 0.013 & - & $0.91(0.86-0.97)$ & 0 & -1 \\
\hline Coagulopathy & $90,551(3.0)$ & $9528(13.3)$ & 0.382 & $1.30(1.22-1.40)$ & $2.12(2.07-2.18)$ & 3 & 9 \\
\hline Obesity & $68,155(2.3)$ & $1011(1.4)$ & 0.065 & $0.64(0.53-0.77)$ & $0.59(0.56-0.63)$ & -4 & -6 \\
\hline Weight loss & $98,545(3.3)$ & $9527(13.3)$ & 0.369 & $1.85(1.67-2.04)$ & $1.67(1.63-1.71)$ & 6 & 6 \\
\hline Fluid and electrolyte disorders & $257,618(8.7)$ & $17,440(24.4)$ & 0.434 & $1.61(1.53-1.69)$ & $1.58(1.55-1.61)$ & 5 & 5 \\
\hline Blood loss anaemia & $19,759(0.7)$ & $685(1.0)$ & 0.033 & $0.81(0.70-0.93)$ & $0.66(0.60-0.71)$ & -2 & -5 \\
\hline Deficiency anaemia & $72,290(2.4)$ & $1886(2.6)$ & 0.013 & $0.80(0.71-0.90)$ & $0.54(0.51-0.56)$ & -2 & -7 \\
\hline Alcohol abuse & $96,708(3.2)$ & $3086(4.3)$ & 0.056 & - & $0.75(0.72-0.78)$ & 0 & -3 \\
\hline Drug abuse & $38,044(1.3)$ & $583(0.8)$ & 0.045 & $0.50(0.42-0.60)$ & $0.67(0.61-0.73)$ & -7 & -5 \\
\hline Psychoses & $29,598(1.0)$ & $404(0.6)$ & 0.049 & - & $0.72(0.65-0.79)$ & 0 & -4 \\
\hline Depression & $173,898(5.8)$ & $3715(5.2)$ & 0.028 & $0.73(0.67-0.80)$ & $0.73(0.70-0.75)$ & -3 & -3 \\
\hline
\end{tabular}

The total cohort percentages can exceed $100 \%$, as each admission contributes to one or more comorbidities. Swiss weights are calculated by dividing the coefficient of each comorbidity by the coefficient in the model with the smallest absolute value (which is 'diabetes uncomplicated' with a coefficient of 0.084 ) and rounding to the nearest whole number

Abbreviations: SMD standardized mean difference between alive and mortality cohort, $V W^{a}$ van Walraven, '-` excluded in the final model, ${ }^{b}$ Row percentage

\section{Discussion}

This study used a six-year dataset of a multi-millionpatient population to explore Charlson and Elixhauser comorbidities with different weightings to predict inhospital mortality. We first derived a set of Swiss weightings for the 31 Elixhauser comorbidities using the national inpatient dataset. The analysis confirmed Charlson and Elixhauser comorbidities with van
Walraven's weights performance for mortality prediction, while the newly derived Swiss weightings slightly improved the mortality prediction for the 31 Elixhauser comorbidities.

Although, the optimized Swiss weightings performed only slightly better than the Charlson and Elixhauservan Walraven sets they also supplied weights for eight Elixhauser comorbidities (e.g. diabetes, hypertension, 


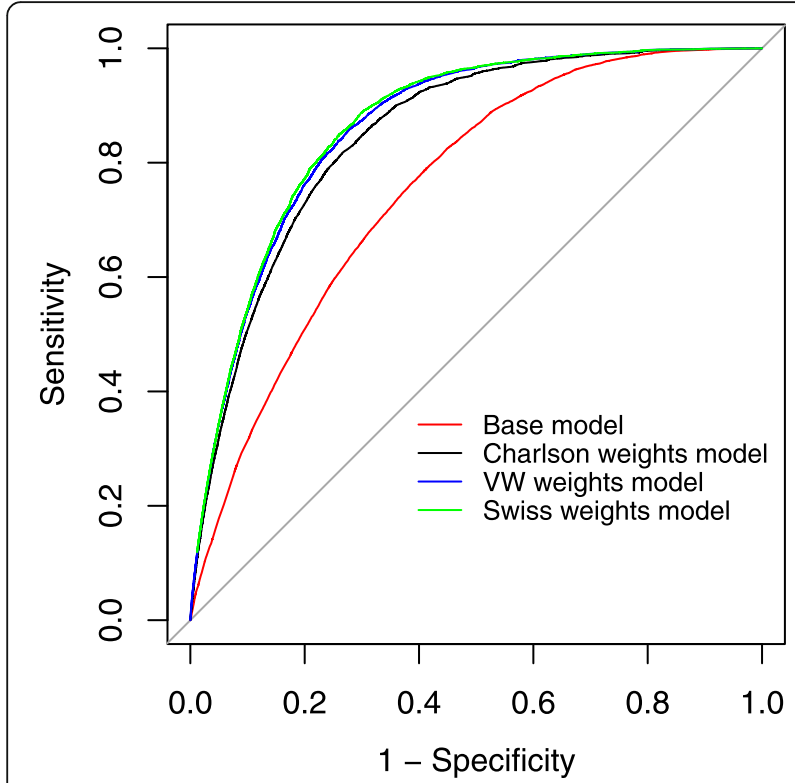

Fig. 1 Receiver-operating characteristic (ROC) curves for generalized additive models predicting in-hospital mortality. Base model (AUC 0.757): age group, sex, hospital types; Charlson weights model (AUC 0.850): base and Charlson weights; WW weights model (AUC 0.863): base and Elixhauser/ van Walraven weights; Swiss weights model (AUC 0.867): base and Elixhauser/ Swiss weights. Straight diagonal line in the middle showing null model (AUC 0.500)

and psychosis) eliminated by van Walraven et al. (2009) [25]. Of the risk-associated comorbidities retained in both the van Walraven and the Swiss weights, several comorbidities showed similar results, e.g., the highest odds ratios to metastatic cancer and liver disease. And regarding the comorbidities with negative associations, only small differences were observed between the van Walraven and Swiss weights (e.g., hypothyroidism or obesity were likely to be healthier).
From an epidemiological perspective, overall hospitalization mortality was only $2.3 \%$, but in-hospital mortality is higher in patients with chronic diseases. Chronic diseases such as cancer, heart and liver diseases increase the risk of dying in hospitals, while certain other less severe diseases, (e.g., hypertension, anaemia and hypothyroidism) have a lower risk. This might be due to the relatively higher frequency of less severe diseases and some reported along with other acute conditions for the same patients. Furthermore, the interpretation of the algebraic sign of a single coefficient from such a joint model is mainly for the derivation of the weights, especially negative weights do not support the survival of the patients. These results are in line with those of Zellweger et al.'s [44] study using the Swiss national death registry of hospital inpatient data from 2010 to 2012. Furthermore, van Walraven et al.'s [25] study based on a single Canadian hospital's records and Thompson et al., [21] using Maryland State inpatient data, showed similar results. These relations could insight the global burden of in-hospital mortality is due to rising chronic diseases.

The existing weighting systems $[11,13,21,25]$ represent data from a specific geographical region, patient group, or even limited numbers of hospitals or settings, matching the generalizability of these weighting systems remained difficult. As this study addresses such issues, with a large dataset representing the Swiss inpatient population, it provides Swiss comorbidity adjustments for the prediction of mortality or other health outcomes. The c statistics reported in our study (weighted models) are around $10 \%$ higher than those reported in van Walraven's study [25]. Several reasons might explain this increase: the GAM modelling approach (with binomial family) including random effects contributing around $2 \%$ improvement in c statistic without random effect, the

Table 3 Comparison of Swiss weights model with Base, Charlson and WW weights models based on the Net Reclassification Improvement (NRI)

\begin{tabular}{|c|c|c|c|c|c|}
\hline \multicolumn{6}{|l|}{ Derivation group } \\
\hline Comparison models & NRI $(95 \% \mathrm{Cl})$ & $\begin{array}{l}\text { Mortality increased } \\
\operatorname{Pr}(U p \mid \text { Case) }\end{array}$ & $\begin{array}{l}\text { Alive increased } \\
\operatorname{Pr}(\text { Up|Ctrl) }\end{array}$ & $\begin{array}{l}\text { Mortality } \\
\text { decreased } \\
\operatorname{Pr} \text { (Down|Case) }\end{array}$ & $\begin{array}{l}\text { Alive } \\
\text { decreased } \\
\operatorname{Pr}(\text { Down|Ctrl) }\end{array}$ \\
\hline $\begin{array}{l}\text { Swiss weights vs. Base } \\
\text { model }\end{array}$ & $0.355(0.352-0.357)$ & $0.448(0.445-0.450)$ & $0.074(0.074-0.074)$ & $0.134(0.133-0.136)$ & $0.115(0.115-0.116)$ \\
\hline $\begin{array}{l}\text { Swiss weights vs. } \\
\text { Charlson weights model }\end{array}$ & $0.049(0.044-0.052)$ & $0.297(0.294-0.299)$ & $0.058(0.058-0.059)$ & $0.251(0.250-0.253)$ & $0.062(0.061-0.062)$ \\
\hline $\begin{array}{l}\text { Swiss weights vs. WW } \\
\text { weights model }\end{array}$ & $0.016(0.013-0.020)$ & $0.157(0.155-0.159)$ & $0.021(0.021-0.022)$ & $0.143(0.140-0.145)$ & $0.023(0.023-0.024)$ \\
\hline
\end{tabular}

Abbreviations: NRI Net Reclassification Improvement with classification cut-off $0.023, \mathrm{Cl}$ confidence interval

$\operatorname{Pr}($ Up, Down) | (Case, Ctrl) represents the proportion of patients whose predicted probabilities increased or decreased for in-hospital mortality and alive cohorts respectively

$\mathrm{NRI}=(\operatorname{Pr}(\mathrm{Up} \mid$ Case $)-\operatorname{Pr}($ Down $\mid$ Case $))+(\operatorname{Pr}($ Down $\mid$ Ctrl $)-\operatorname{Pr}(\mathrm{Up} \mid \mathrm{Ctrl}))$

Base model: age group, sex, hospital types

Charlson weights model: base and Charlson weights

VW weights model: base and Elixhauser/ van Walraven weights

Swiss weights model: base and Elixhauser/ Swiss weights 
study cohort and hospital types included might raise the base model and largely the conditional interpretation of weightings effect raised c statistic of weighted models. With the new eight derivations, the additional eight significant variables might have played a role too. However, a slightly improved performance of the Swiss weights system suggests that it might be worthwhile to derive country- or region-specific comorbidity weights from representative patient populations.

C-statistics and ROCs are widely used to assess predictive performance. Nonetheless, one downside of comparing c-statistic and ROCs is that differences between c-statistics are often small, [45] as it was the case when we compared our new weights and van Walraven's. Over the past decade, it has become common to use NRIs to compare different models' performance, even though it might differ with the cut-offs taken for analysis $[39,46]$. In our study, taking the same cut-offs for all models, NRI calculations confirmed the three weighting systems' rankings i.e., Swiss, van Walraven and Charlson weights.

The primary strength of this study was the large sample size and the heterogeneity of the Swiss inpatient population across all general hospitals over 6 years, which made it representative of the entire country. To our knowledge, this study is the first to derive and validate Elixhauser weightings in Swiss hospital inpatient data. We used standard regression methodology for large datasets, including random effects at the hospital level, and internally validated our models. We also used accepted methods to modify our adjusted model into a Swiss weightings system that re-includes the association of several comorbidities (e.g., diabetes, hypertension, Psychoses) formerly excluded from the Elixhauser index in the VW study [35]. Despite differences in individual comorbidities' prevalence and weightings, Charlson, Elixhauser/VW, and the Swiss weights performed well across the derivation, validation, and all-cases groups. We also used NRIs, allowing a robust comparison of model performance. Finally, the methods we applied were explicit and can be replicated by other researchers, who can adjust or control for patient comorbidity via their hospital and national databases. Moreover, the managerial utility could be done using this method by identifying high-risk patients for safe care and by evaluating hospitals performance based on the patient's outcome.

Our study also has certain notable limitations. We derived our weights using statistical criteria, while clinical knowledge might be needed to determine each comorbidity's value. Since we used codes assigned in routine data, the capture of the comorbidities could be influenced by other factors, such as physician and nurse documentation, code assignment accuracy, and the possibility that capture of comorbidities is biased towards those for which the Swiss DRG / MDC pays more [43, 47]. The negative coefficients/weights might be artefacts, as they are computed using routine data and coding of these is influenced by the main diagnose (e.g., deficiency anaemia, diabetes or hypertension are far more likely to be recorded when a patient had few other serious or acute problems). The direction of the coefficients is also driven by the joint adjusted model, which makes the interpretation of a single coefficient not meaningful. Moreover, some researchers believe current comorbidity indices are not suitable for use as predictors of patientcentred outcomes like rehabilitation, readmission, feefor-services while weightings might differ in restricted cohorts, other outcomes and countries [27]. Additionally, Swiss data protection regulations prevented us from obtaining the inpatients' exact age, we could not differentiate children exactly under 18 years and could not specify each year. This also might have influenced the predictive accuracy of the tested models.

\section{Conclusions}

We found that Elixhauser/van Walraven weightings performed well in a large Swiss dataset and could derive Swiss weightings with statistically significant, yet with a small improvement in mortality prediction. Although the Swiss weightings showed slightly improved mortality predictions, we confirmed the validity of the Elixhauser/ van Walraven weightings. The results provide evidence that Elixhauser/van Walraven weightings continue to be the preferred choice for weighting. In the Swiss context and possibly in countries with ICD-10 GM (German Modification) the derived weights are an option and to identify high-risk patients for safe care/treatment. Given access to similar data, researchers could use the methods described here to validate existing weightings such as van Walraven or derive their own country- or regionspecific morbidity weights, although improvements might be small.

\section{Supplementary Information}

The online version contains supplementary material available at https://doi. org/10.1186/s12913-020-05999-5.

\section{Additional file 1.}

\section{Abbreviations}

ECl: Elixhauser Comorbidity Index; CCl: Charlson Comorbidity Index; ICD - 10 GM: International Classification of Diseases version-10 German Modification; DRGs: Diagnosis-Related Groups; SMD: Standardized Mean Differences; NRI: Net Reclassification Improvement; MDCs: Major Diagnostic Categories; ROC: Receiver-Operating Characteristic; FSO: Federal Statistical Office; SBK: Swiss Nurses' Association; GAM: Generalized Additive Model; FMH: Swiss Medical Association; Cl: Confidence Interval

\section{Acknowledgements}

This paper is derived from the PhD dissertation research in the field of health service and patient safety of the first author. We would like to thank the 
Swiss Federal Statistics Office (FSO) and its data management team for their support in providing inpatient data for our analyses. We are especially grateful to all the Swiss hospitals for their regular data reporting and information forwarded to the FSO and we would like to thank Mr. Chris Shultis for English editing of this paper. We are also thankful to the Swiss Nurses' Association (SBK) for their financial support for this study.

\section{Authors' contributions}

NS and MS had full access to all of the data in the study and take responsibility for the integrity of the data and accuracy of the analysis. NS, RS, OE, DA, and MS contributed to the conception and design of the study. NS drafted the manuscript and all the authors contributed substantially to the interpretation, visualization of the data, critically revised, edited the manuscript for important intellectual content and agreed to be accountable for all aspects. All of the authors listed above approved this version of the manuscript to be published.

\section{Funding}

This study was supported by Swiss Nurses' Association (SBK). The funders had no role in the design, conduct, analysis or reporting of this study.

\section{Availability of data and materials}

Upon application, the data that support the findings of this study are available from Federal Statistical Office (FSO), Switzerland.

\section{Ethics approval and consent to participate}

Data access was granted by the Swiss Federal Statistics Office based on a data protection contract as stipulated by article 22 of the Swiss Federal Act on Data Protection. The data is routinely collected and de-identified and therefore exempt from further ethical approval. Consent to participate was not applicable.

\section{Consent for publication}

Not applicable.

\section{Competing interests}

The authors declare that they have no competing interests.

\section{Author details}

'Institute of Nursing Science (INS), Department Public Health (DPH), Faculty of Medicine, University of Basel, Basel, Switzerland. ${ }^{2}$ Patient Safety Office, University Hospital Basel, Basel, Switzerland. ${ }^{3}$ Directorate of Medicine, Inselspital University Hospital Bern, Bern, Switzerland. ${ }^{4}$ College of Health-Care Professions Claudiana, Bozen, Italy. ${ }^{5}$ Nursing Research Unit, Inselspital University Hospital Bern, Bern, Switzerland.

\section{Received: 26 June 2020 Accepted: 8 December 2020}

Published online: 06 January 2021

\section{References}

1. Harrison C, Britt H, Miller G, Henderson J. Examining different measures of multimorbidity, using a large prospective cross-sectional study in Australian general practice. BMJ Open. 2014;4(7):e004694.

2. Pefoyo AJK, Bronskill SE, Gruneir A, et al. The increasing burden and complexity of multimorbidity. BMC Public Health. 2015;15(1):415.

3. Metcalfe D, Masters J, Delmestri A, et al. Coding algorithms for defining Charlson and Elixhauser co-morbidities in read-coded databases. BMC Med Res Methodol. 2019;19(1):115.

4. Wallace E, Salisbury C, Guthrie B, Lewis C, Fahey T, Smith SM. Managing patients with multimorbidity in primary care. Bmj. 2015;350:h176.

5. Barnett K, Mercer SW, Norbury M, Watt G, Wyke S, Guthrie B. Epidemiology of multimorbidity and implications for health care, research, and medical education: a cross-sectional study. Lancet. 2012;380(9836):37-43.

6. Fraccaro $\mathrm{P}$, Kontopantelis $\mathrm{E}$, Sperrin $\mathrm{M}$, et al. Predicting mortality from change-over-time in the Charlson Comorbidity Index: A retrospective cohort study in a data-intensive UK health system. Medicine. 2016;95(43):e4973.

7. Moore BJ, White S, Washington R, Coenen N, Elixhauser A. Identifying increased risk of readmission and in-hospital mortality using hospital administrative data. Med Care. 2017:55(7):698-705.
8. Divo $\mathrm{M}$, Cote $\mathrm{C}$, de Torres JP, et al. Comorbidities and risk of mortality in patients with chronic obstructive pulmonary disease. Am J Respir Crit Care Med. 2012;186(2):155-61.

9. Toson B, Harvey LA, Close JC. The ICD-10 Charlson comorbidity index predicted mortality but not resource utilization following hip fracture. J Clin Epidemiol. 2015;68(1):44-51.

10. Elixhauser A, Steiner C, Harris DR, Coffey RM. Comorbidity measures for use with administrative data. Med Care. 1998:8-27.

11. Charlson ME, Pompei $P$, Ales $K L$, Mackenzie CR. A new method of classifying prognostic comorbidity in longitudinal studies: development and validation. J Chronic Dis. 1987:40(5):373-83.

12. Chang H-J, Chen P-C, Yang C-C, Su Y-C, Lee C-C. Comparison of Elixhauser and Charlson methods for predicting oral cancer survival. Medicine. 2016; 95(7):e2861

13. Menendez ME, Neuhaus V, Van Dijk CN, Ring D. The Elixhauser comorbidity method outperforms the Charlson index in predicting inpatient death after orthopaedic surgery. Clin Orthopaedics Related Research ${ }^{\oplus}$. 2014;472(9): 2878-86.

14. Fortin Y, Crispo JA, Cohen D, McNair DS, Mattison DR, Krewski D. External validation and comparison of two variants of the Elixhauser comorbidity measures for all-cause mortality. PLoS One. 2017:12(3):e0174379.

15. Dailiana Z, Papakostidou I, Varitimidis S, Michalitsis S, Veloni A, Malizos K. Surgical treatment of hip fractures: factors influencing mortality. Hippokratia. 2013;17(3):252.

16. Shetty KD, Bhattacharya J. Changes in hospital mortality associated with residency work-hour regulations. Ann Intern Med. 2007;147(2):73-80.

17. Southern DA, Quan H, Ghali WA. Comparison of the Elixhauser and Charlson/Deyo methods of comorbidity measurement in administrative data. Med Care. 2004;42(4):355-60.

18. Gasparini A. comorbidity: An R package for computing comorbidity scores. Open Source Software. 2018;3(23):648

19. Quan H, Sundararajan V, Halfon $P$, et al. Coding algorithms for defining comorbidities in ICD-9-CM and ICD-10 administrative data. Med Care. 2005; 43(11):1130-9.

20. Te Grotenhuis M, Pelzer B, Eisinga R, Nieuwenhuis R, Schmidt-Catran A, Konig R. When size matters: advantages of weighted effect coding in observational studies. Int J Public Health. 2017;62(1):163-7.

21. Thompson NR, Fan Y, Dalton JE, et al. A new Elixhauser-based comorbidity summary measure to predict in-hospital mortality. Med Care. 2015;53(4):374.

22. Pavlou M, Ambler G, Seaman SR, et al. How to develop a more accurate risk prediction model when there are few events. Bmj. 2015;351:h3868.

23. Song M-K, Lin F-C, Ward SE, Fine JP. Composite variables: when and how. Nurs Res. 2013;62(1):45.

24. Pule ML, Buckley E, Niyonsenga T, Roder D. The effects of comorbidity on colorectal cancer mortality in an Australian cancer population. Sci Rep. 2019; $9(1): 8580$

25. van Walraven $\mathrm{C}$, Austin PC, Jennings A, Quan $\mathrm{H}$, Forster AJ. A modification of the Elixhauser comorbidity measures into a point system for hospital death using administrative data. Med Care. 2009:47(6):626-33.

26. Austin SR, Wong Y-N, Uzzo RG, Beck JR, Egleston BL. Why summary comorbidity measures such as the Charlson comorbidity index and Elixhauser score work. Med Care. 2015;53(9):e65.

27. Kumar A, Karmarkar AM, Graham JE, et al. Comorbidity indices versus function as potential predictors of 30-day readmission in older patients following postacute rehabilitation. J Gerontol Series A: Biomedical Sciences and Medical Sciences. 2017;72(2):223-8.

28. Stanley J, Sarfati D. The new measuring multimorbidity index predicted mortality better than Charlson and Elixhauser indices among the general population. J Clin Epidemiol. 2017;92:99-110.

29. De Pietro C, Camenzind P, Sturny I, et al. Switzerland: health system review. Health Systems Transition. 2015;17(4):1-288.

30. Austin PC. An introduction to propensity score methods for reducing the effects of confounding in observational studies. Multivar Behav Res. 2011; 46(3):399-424.

31. Wilson M, Welch J, Schuur J, O'Laughlin K, Cutler D. Hospital and emergency department factors associated with variations in missed diagnosis and costs for patients age 65 years and older with acute myocardial infarction who present to emergency departments. Acad Emerg Med. 2014;21(10):1101-8.

32. Li Z, Wood SN. Faster model matrix crossproducts for large generalized linear models with discretized covariates. Stat Comput. 2020;30(1):19-25. 
33. Li Z, Wood SN. Faster model matrix crossproducts for large generalized linear models with discretized covariates. Stat Comput. 2019:30(1):1-7.

34. R Core Team. R: a language and environment for statistical computing. Vienna: R Foundation for Statistical Computing; 2019.

35. Sullivan LM, Massaro JM, D'Agostino RB Sr. Presentation of multivariate data for clinical use: the Framingham study risk score functions. Stat Med. 2004; 23(10):1631-60.

36. Quan $\mathrm{H}$, Li B, Couris CM, et al. Updating and validating the Charlson comorbidity index and score for risk adjustment in hospital discharge abstracts using data from 6 countries. Am J Epidemiol. 2011;173(6):676-82.

37. Pencina MJ, D'Agostino RB. Evaluating discrimination of risk prediction models: the C statistic. Jama. 2015;314(10):1063-4.

38. Pencina MJ, D'Agostino RB Sr, D'Agostino RB Jr, Vasan RS. Evaluating the added predictive ability of a new marker: from area under the ROC curve to reclassification and beyond. Stat Med. 2008;27(2):157-72.

39. Pencina MJ, D'Agostino RB Sr, D'Agostino RB Jr, Vasan RS. Comments on 'integrated discrimination and net reclassification improvements-practical advice'. Stat Med. 2008;27(2):207-12

40. Pencina MJ, D'Agostino RB Sr, Steyerberg EW. Extensions of net reclassification improvement calculations to measure usefulness of new biomarkers. Stat Med. 2011;30(1):11-21.

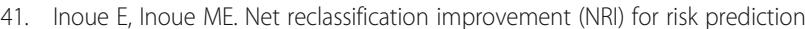
models with time to event and binary response data; 2018.

42. Gagne JJ, Glynn RJ, Avorn J, Levin R, Schneeweiss S. A combined comorbidity score predicted mortality in elderly patients better than existing scores. J Clin Epidemiol. 2011:64(7):749-59.

43. Wasserfallen J-B, Zufferey J. Financial impact of introducing the Swiss-DRG reimbursement system on potentially avoidable readmissions at a university hospital. Swiss Med Wkly. 2015;145:w14097.

44. Zellweger U, Junker C, Bopp M, Group SNCS. Cause of death coding in Switzerland: evaluation based on a nationwide individual linkage of mortality and hospital in-patient records. Popul Health Metrics. 2019;17(1):2.

45. Loging WT. Bioinformatics and computational biology in drug discovery and development: Cambridge University press; 2016.

46. Leening MJ, Vedder MM, Witteman JC, Pencina MJ, Steyerberg EW. Net reclassification improvement: computation, interpretation, and controversies: a literature review and clinician's quide. Ann Intern Med. 2014;160(2):122-31.

47. Quan H, Eastwood C, Cunningham CT, et al. Validity of AHRQ patient safety indicators derived from ICD-10 hospital discharge abstract data (chart review study). BMJ Open. 2013;3(10):e003716.

\section{Publisher's Note}

Springer Nature remains neutral with regard to jurisdictional claims in published maps and institutional affiliations.

Ready to submit your research? Choose BMC and benefit from:

- fast, convenient online submission

- thorough peer review by experienced researchers in your field

- rapid publication on acceptance

- support for research data, including large and complex data types

- gold Open Access which fosters wider collaboration and increased citations

- maximum visibility for your research: over $100 \mathrm{M}$ website views per year

At $\mathrm{BMC}$, research is always in progress.

Learn more biomedcentral.com/submissions 